\title{
Pet rats as a source of hantavirus in England and Wales, 2013
}

L J Jameson (lisa.jameson@hpa.org.uk) ${ }^{1,2}$, S K Taori³, B Atkinson ${ }^{1}$, P Levick ${ }^{4}$, C A Featherstone ${ }^{5}$, G van der Burgt ${ }^{6}$, N McCarthy ${ }^{7}$, J Hart ${ }^{8}$, J C Osborne ${ }^{3}$, A L Walsh ${ }^{9}$, T J Brooks ${ }^{3}$, R Hewson ${ }^{1}$

1. Virology and Pathogenesis, Microbiology Services, Health Protection Agency, Porton Down, Wiltshire, United Kingdom

2. Department of Clinical Infection, Microbiology and Immunology, Institute of Infection and Global Health, University of Liverpool, Liverpool, United Kingdom

3. Rare and Imported Pathogens Department, Microbiology Services, Health Protection Agency, Porton Down, Wiltshire, United Kingdom

4. Biological Investigations Group, Microbiology Services, Health Protection Agency, Porton Down, Wiltshire, United Kingdom

5. Animal Health and Veterinary Laboratories Agency, Thirsk, Yorkshire, United Kingdom

6. Animal Health and Veterinary Laboratories Agency, Luddington, Warwickshire, United Kingdom

7. Thames Valley Health Protection Unit, Centre for Radiation, Chemical \& Environmental Hazards, Health Protection Agency, Oxfordshire, United Kingdom

8. Public Health Wales, Health Protection Team, Flintshire, United Kingdom

9. Gastrointestinal, Emerging and Zoonotic Infections Department, Health Protection Services, Health Protection Agency Colindale, London, United Kingdom

Jameson LJ, Taori SK, Atkinson B, Levick P, Featherstone CA, van der Burgt G, McCarthy N, Hart J, Osborne JC, Walsh AL, Brooks TJ, Hewson R. Pet rats as a source of hantavirus in England and Wales, 2013. Euro Surveill. 2013;18(9):pii=20415. Available online: http://www.eurosurveillance.org/ViewArticle.aspx?Articleld=20415

Article submitted on 21 February 2013 / published on 28 February 2013

We report the detection of a strain of Seoul hantavirus (SEOV) in pet rats in England and Wales. The discovery followed an investigation of a case of haemorrhagic fever with renal syndrome in Wales. Hantavirus RNA was detected via real-time reverse transcription-polymerase chain reaction (RT-PCR) and classic RT-PCR in pet rats belonging to the patient. Sequencing and phylogenetic analysis confirmed the virus to be a SEOV that is similar, but not identical, to a previously reported United Kingdom strain from wild rats.

In January 2013, a male patient in north Wales suffering from acute kidney injury and clinically presenting with haemorrhagic fever and renal syndrome, tested seropositive (IgG 1:10,000) for Seoul (SEOV) and Hantaan (HTNV) hantavirus using indirect immunofluorescence (Euroimmun, Germany). A previous blood sample from October 2012, taken for unrelated purposes, was retrospectively obtained and tested. This sample demonstrated no antibody to hantavirus thus confirming serconversion as defined by Heyman et al., 2007 [1]. Epidemiological assessment identified the patient's two pet agouti rats (Rattus norvegicus) as a possible source of the hantavirus.

\section{Virus investigation}

Due to the patient's serious clinical condition the pet rats were in the care of the original breeder in Oxfordshire, south England. They were housed in a separate building to that of the breeder's pet rat colony. Blood samples from both rats, and urine from one of the two rats, were obtained and processed for RNA extraction using RNeasy kit (Qiagen). The extract was tested using a modified version of a previously published realtime reverse transcription-polymerase chain reaction
(RT-PCR) assay for the dual detection of HTNV and SEOV [2]. The modifications adopted were: (i) use of a single Minor Groove Binder (MGB) -probe with a degenerate single base change (5' FAM-TCAATGGGRATACAACT-3') in place of the two non-degenerate published MGBprobes and (ii) use of the SuperScript III Platinum Onestep qRT-PCR kit (Invitrogen) in accordance with the manufacturer's specifications. In-house validation confirmed that these changes had no detrimental impact on assay sensitivity and reduced the cost of the test.

The urine and blood samples tested positive and results were rapidly fed back to the incident control team, which included representatives from Health Protection Agency (HPA, Porton and Colindale), Thames Valley Health Protection Unit (HPU), Public Health Wales (PHW), Environmental Health (EH) and Animal Health and Veterinary Laboratories Agency (AHVLA). Due to uncertainties regarding the prevalence of this virus within the pet rat population in the United Kingdom (UK), the nature of its transmission, and the potential seriousness of human disease in this particular instance, the owner's consent was obtained to euthanase the two pet rats and remove them for further testing at the HPA Porton. One of the rats was processed as previously described [3] and viral RNA sourced directly from lung tissue was subjected to additional characterisation of the virus through standard Sanger sequencing on a 3130xl sequencer (Life Technologies). Sequencing of the $\mathrm{S}$ segment was achieved, confirming the virus was indeed a strain of SEOV similar, but not identical, to previously isolated UK SEOV strains: Humber (wild rats) [3] and IR461 (laboratory rats) [4]. We have provisionally designated this strain 'Cherwell'. Alignments of sequences from the virus strains were 
Phylogenetic analysis of S segment sequence of the Seoul hantavirus Cherwell strain derived from a pet rat, England, February 2013

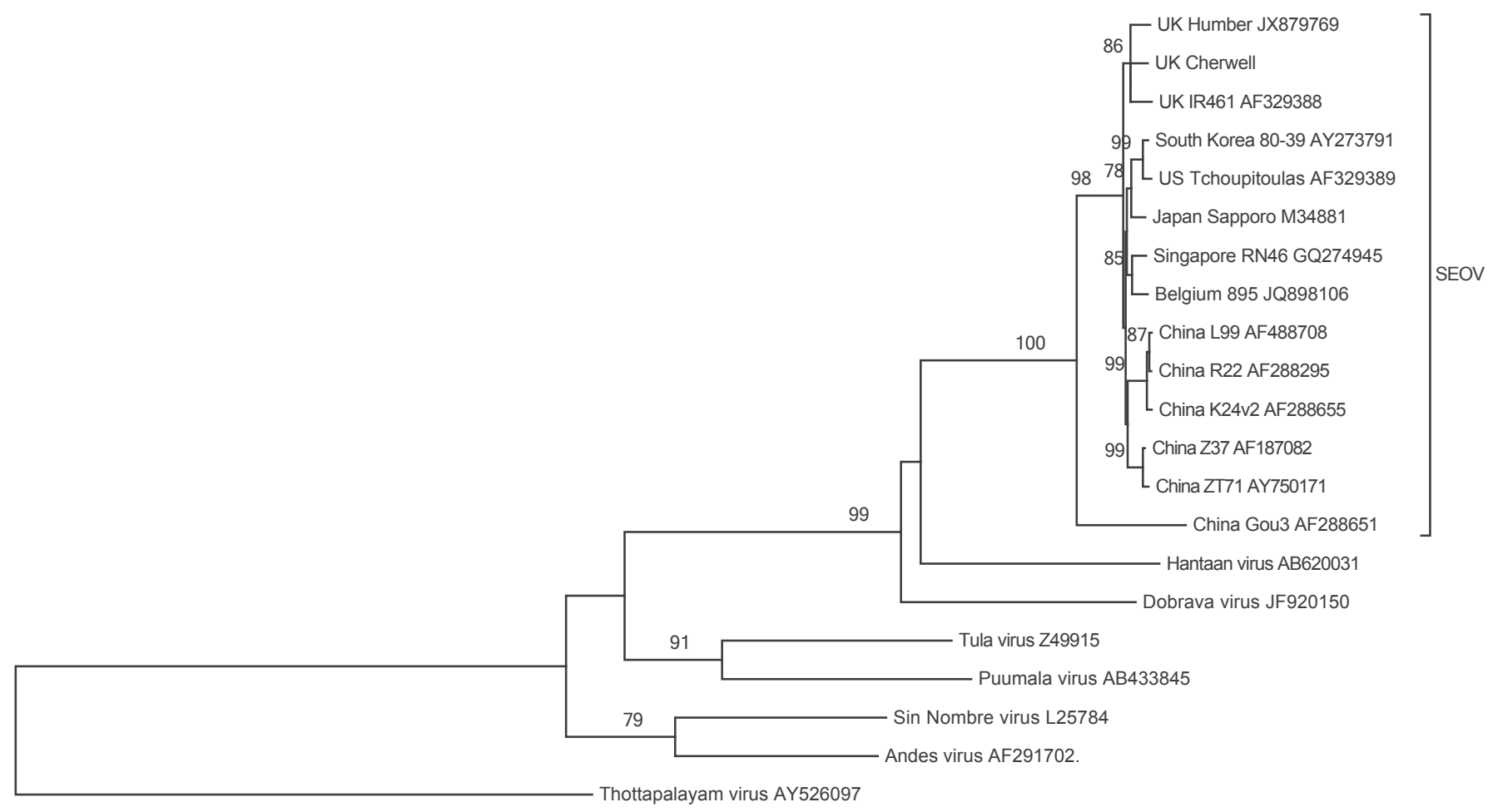

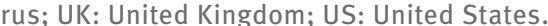

Horizontal distances represent the number of nucleotide differences. Bootstrap confidence limits exceeding $70 \%$ are shown for each branch node. The phylogenetic tree was based on S segment sequences of previously published SEOV strains and other major hantavirus species as well as the sequence derived from the pet rat (Rattus norvegicus). The available geographical origin of the viral sequences, the viruses' names and respective GenBank accession numbers figure on the tree.

conducted using ClustalW and the molecular evolutionary genetics analysis (MEGA5) programme suite [5] was used to perform phylogenetic analysis. Comparisons between Cherwell and Humber $S$ segments highlighted a total of 47 nucleotide differences, 36 within the open reading frame (ORF) resulting in one amino acid difference (methionine to isoleucine) at position 247. Phylogenetic analysis using the neighbour-joining method in MEGA5, with bootstrap values $(2,000$ replicates), placed the Cherwell $S$ segment within the same group as the corresponding segments of Humber and IR461 (Figure). The sequence ( 1,769 nucleotides) was released to GenBank under accession number KC626089.

Following confirmation of hantavirus infection in these two rats, blood samples were obtained with the owner's permission, from 21 of the breeding colony rats and processed in the same way. Guidance was provided to the breeder to minimise the potential risk of infection while caring for the remaining rats, should they be positive. On the day of sampling, 7/21 rats had detectable RNA specific to HTNV/SEOV in blood. Preliminary sequence data indicates the same Cherwell strain in these rats.

\section{Investigation of human contacts with rats}

A blood sample was obtained from the patient's partner in Wales; this was negative for IgG antibodies. Blood samples were also obtained from the breeder and her spouse in England. The breeder, who had most contact with the rats in the breeding colony, had a low positive titre to HTNV and SEOV (IgG 1: 100). The breeder's spouse tested strongly positive with an IgG titre of 1: 10,000 to HTNV and SEOV, strongly suggesting hantavirus infection. Retrospective investigation of his medical records showed that he had been admitted to hospital in late 2011 with an undiagnosed viral illness resulting in acute renal impairment and thrombocytopenia. It is now considered highly likely that this was due to hantavirus infection; an archived blood sample from this admission was retrospectively tested 
and demonstrated an IgG titre of 1: 1,000 to HTNV and SEOV. Detailed clinical findings of these cases will be published shortly [6]. In summary, of four people exposed to this particular population of rats, two had been clinically ill with renal impairment and were strongly seropositive, one had a low level of antibody with no clinical illness, and one was seronegative.

\section{Control measures}

Transmission of hantaviruses to humans most often occurs through breathing in aerosols of excreta from infected rodents [7]. Large quantities of infectious virus are intermittently excreted in the urine, saliva and faeces of infected rodents. The unique finding in this investigation of a strain of SEOV in pet rats, rather than wild rats, posed a challenge for infection control and involved a multi-disciplinary panel including medical/scientific experts from HPA, HPU, PHW, EH, and veterinarians from AHVLA. As part of this investigation the pet rats were euthanised, with owner's consent, in order to further scientific understanding of hantavirus infection in pet rats. Recommendations for management of any future incidents would be made on a case for case basis. Further studies are planned to gather evidence on the prevalence of this virus in the pet rat community which will inform future risk assessment and the provision of appropriate public health guidance. Interim guidance on minimising the infection to the pet rat community has been prepared [8] and will continue to be updated as the investigation progresses.

\section{Discussion and conclusions}

In January 2013, we reported the isolation of a UK strain of SEOV (Humber) from wild rats in north-east England [3]. We now report a second SEOV strain from the UK which is similar, but genetically distinct from the Humber isolate and laboratory-associated IR461 isolate. Further research will continue to investigate the relationship of these three strains. The pet rats identified in this investigation are part of a wider pet rat community which partakes in national and international shows and fosters international sharing of rats. Currently, the prevalence of SEOV in the UK pet rat community is unknown, but if SEOV infection was widespread, there would be implications for the wider (non-UK) pet rat community. The HPA is continuing to investigate this newly recognised source of hantavirus infection in collaboration with the AHVLA. Should overall findings indicate that further health protection advice is necessary, the HPA will work with the relevant partners to provide this.
Acknowledgements

The authors would like to thank the rat owners for their cooperation and acknowledge, the input of Daniel Rowlson from Cherwell District Council for his role in the investigation.

\section{Funding}

This work is produced by L.J Jameson under the terms of Doctoral research training fellowship issued by the NIHR. $R$ Hewson and $B$ Atkinson also acknowledge the funding of NIHR for emerging diseases. The veterinary support for the investigation was funded by Defra as part of project FZ2100: Surveillance for Non-statutory Zoonoses.

Conflicts of interest

None declared.

Authors' contributions

Lisa Jameson, Barry Atkinson and Roger Hewson were responsible for the virological analysis and the interpretation of laboratory results. Surabhi Taori, Jane Osborne and Tim Brooks coordinated the national public health response and were responsible for clinical diagnosis. Peter Levick, Charlotte Featherstone and Guda van der Burgt performed the blood sampling. Noel McCarthy and Judy Hart coordinated the local public health response. Amanda Walsh contributed to the national public health response and prepared public health guidance for dissemination. Lisa Jameson wrote the draft manuscript and all authors revised and approved the final version.

\section{References}

1. Heyman P, Cochez C, Ducoffre G, Mailles A, Zeller H, Abu Sin $M$, et al. Haemorrhagic Fever with Renal Syndrome: an analysis of the outbreaks in Belgium, France, Germany, the Netherlands and Luxembourg in 2005. Euro Surveill. 2007;12(5):pii=712. Available from: http://www.eurosurveillance.org/ViewArticle. aspx?Articleld $=712$

2. Kramski M, Meisel H, Klempa B, Krüger DH, Pauli G, Nitsche A. Detection and typing of human pathogenic hantaviruses by real-time reverse transcription-PCR and pyrosequencing. Clin Chem. 2007:53(11):1899-905. http://dx.doi.org/10.1373/ clinchem.2007.093245 PMid:17717126

3. Jameson LJ, Logue CH, Atkinson B, Baker N, Galbraith SE, Carroll MW, et al. The continued emergence of hantaviruses: isolation of a Seoul virus implicated in human disease, United Kingdom, October 2012. Euro Surveill. 2013;18(1): $\mathrm{pii}=20344$. Available from: http://www.eurosurveillance.org/ViewArticle. aspx?Articleld=20344 PMid:23305714

4. Shi X, McCaughey C, Elliott RM. Genetic characterisation of a hantavirus isolated from a laboratory-acquired Infection. J Med Virol. 2003;71(1):105-9. http://dx.doi.org/10.1002/jmv.10446 PMid:12858415

5. Tamura K, Peterson D, Peterson N, Stecher G, Nei M, Kumar S. MEGA5: molecular evolutionary genetics analysis using naximum likelihood, evolutionary distance, and maximum parsimony methods. Mol Biol Evol. 2011;28(10):2731-9. http://dx.doi.org/10.1093/molbev/msr121 PMid:21546353 PMCid:3203626

6. Taori SK, Jameson LI, Campbell A, Drew P, McCarthy N, Hart J, et al. Renal failure: Oh rats.......it's a hantavirus! Lancet. Forthcoming 2013.

7. Krüger DH, Schönrich G, Klempa B. Human pathogenic hantaviruses and prevention of infection. Hum Vaccin. 2011;7(6):685-93. http://dx.doi.org/10.4161/hv.7.6.15197 PMid:21508676 PMCid:3219076

8. Health Protection Agency (HPA). Reducing the risk of infection from pet rodents. 2013. London:HPA; 20 Feb 2013. Available from: http://www.hpa.org.uk/Topics/InfectiousDiseases/ InfectionsAZ/Zoonoses/Generallnformation/ zooo10ZoonosesFromRats/ 